\title{
INSECTOS PLAGAS Y BENÉFICOS ASOCIADOS AL MARAÑÓN (Anacardium occidentale L.) ORGÁNICO Y CONVENCIONAL, EN LEÓN, NICARAGUA
}

\section{INSECT PESTS AND THEIR NATURAL ENEMIES ASSOCIATED TO CASHEW NUT (Anacardium occidentale L.) IN ORGANIC AND CONVENTIONAL FARMING IN LEON, NICARAGUA}

\section{Jiménez-Martínez Edgardo ${ }^{1}$, Gómez-Martínez Jorge ${ }^{2}$}

${ }^{1} \mathrm{PhD}$ en Entomología, Universidad Nacional Agraria, UNA, Profesor Titular, Docente-Investigador.

${ }^{2} \mathrm{MSc}$. en agroecología y desarrollo sostenible, UNA, Profesor adjunto, Docente-Investigador.

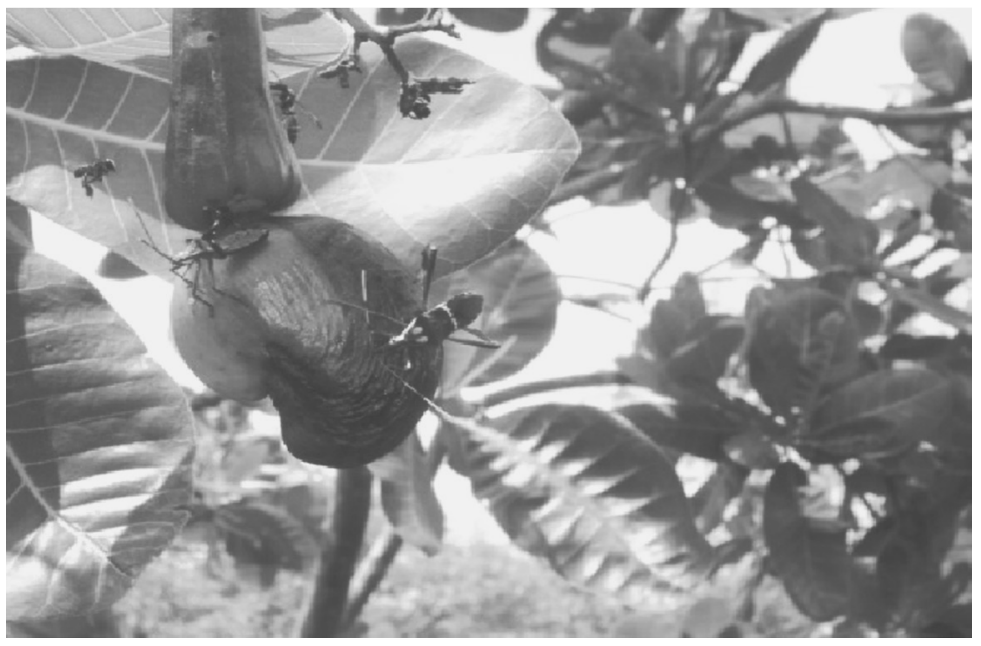

\section{RESUMEN}

El presente estudio se realizó durante el período julio 2009 a marzo del 2010, en la comarca Chacraseca, del departamento de León, El objetivo de este estudio fue identificar y describir la fluctuación poblacional y el rol de los principales insectos plagas y benéficos asociados al marañón orgánico y convencional. Para llevar a cabo este estudio se seleccionaron tres fincas orgánicas y tres fincas convencionales, en cada finca se seleccionó una parcela de $1 \mathrm{mz}$ (0.7 ha), en cada parcela se seleccionaron cinco sitios fijos de muestreos, los muestreos se realizaron semanalmente a través de capturas manuales de insectos plagas e insectos benéficos del follaje. Los insectos colectados en el campo se llevaron al laboratorio de entomología de la Universidad Nacional Agraria para su respectiva identificación. Las variables evaluadas fueron: Número de insectos Homóptera, Heteróptera, Coleóptera, Neuróptera, Díptera, e Hymenóptera por familia. Los resultados fueron los siguientes: se encontró un mayor número de insectos plagas y benéficos en fincas de marañón orgánico que en convencional. Se identificó y se describió las fluctuaciones poblacionales de los insectos miembros de las familias: Coreidae, Cicadellidae, Tenebrionidae, Chrysomelidae, Apidae, Formicidae, Dolichopodidae, y Vespidae asociados a este cultivo, se encontró que las familias de insectos Cicadellidae, Tenebrionidae, Chrysomelidae, Coccinellidae y Formicidae se presentaron con más frecuencia durante la época de crecimiento vegetativo del cultivo, los insectos de la familia Dolichopodidae y Vespidae se presentaron con más frecuencia durante la época de floración del cultivo; y los insectos de la familia Coreidae y Apidae se presentaron con más frecuencia durante la época de fructificación del cultivo.

\section{ABSTRACT}

This study was conducted during the period from July 2009 to March 2010, in Chacraseca, department of León, the aim of this study was to identify and describe the population fluctuations and the role of the principal insect pest and their natural enemies associated to organic and conventional cashew nut plots. To carry out this study, three organic and three conventional farms were selected; in each farm, a plot of $1 \mathrm{mz}$ ( $0.7 \mathrm{ha}$ ) was studied,in each plot, five fixed sampling sites were established, sampling was conducted weekly through insects pests and beneficial insects manual catches on foliage. Field collected insects were brought to the entomology laboratory of the National Agrarian University for their respective identification. The evaluated variables were: Number of insects from the Homoptera, Heteroptera, Coleoptera, Neuroptera, Diptera andHymenopterafamilies. The results were as follow: A higher number of pest and beneficial insects were found in organic than in conventional farms.It was identified and described the population fluctuations of the members of the insect families: Coreidae, Cicadellidae, Tenebrionidae, Chrysomelidae, Apidae, Formicidae, Dolichopodidaeand Vespidae associated to cashew nut. In addition, it was found thatinsect families such as Cicadellidae, Tenebrionidae, Chrysomelidae, Coccinellidae and Formicidae occurred more frequently during the period of vegetative growth of the crop.Besides, insects of the family Dolichopodidaeand Vespidae occurred more frequently during flowering of the crop; and finally, insects of the family Coreidae and Apidae occurred more frequently during fruiting of the crop. 
$\mathrm{E}$ 1 marañón (Anacardium occidentale L.) es un árbol de la familia de las Anacardiácea, se cultiva en zonas con clima tropical y sub tropical. A nivel internacional se conocen 18 variedades destacándose las variedades manzana amarilla y manzana roja como las más comunes. Entre los géneros y especies más cultivadas están: $A$. mericanum, $A$. indicum, $A$. occidentale (Jaramillo, 2003).

Actualmente los mayores productores mundiales de la nuez de marañón son: la India, Brasil, Vietnam, Mozambique y Tanzania. En el 2003 el cultivo alcanzó una producción mundial aproximada de 1, 200,000 t de semilla con un valor de mercado cercano a los 1500 millones de dólares (Cuenta reto del milenio, 2007).En Nicaragua, la semilla de marañón comenzó a tener importancia comercial en el año 1972 y en la actualidad ha adquirido mayor demanda a nivel nacional e internacional, ya que es considerado como un cultivo de mucha importancia económica para la zona del occidente del país (Membreño, 2002).

El cultivo del marañón se ha estado sembrando de forma comercial, en las zonas del Occidente de Nicaragua (León y Chinandega) desde hace varios años especialmente en las comunidades de Chacraseca, Lechecuagos, Las Lomas, El Espino y Larreynaga. En la comarca Chacraseca, la siembra y aprovechamiento de este cultivo se ha venido realizando en pequeñas parcelas tanto convencionales como orgánicas, esto se debe a la demanda, rentabilidad y la importancia de este cultivo a nivel internacional (CIPRES, 2008).

Según Gliessman, (1998) los objetivos de la agricultura convencional es maximizar la producción y las ganancias, sin embargo para alcanzar estos objetivos se han desarrollado prácticas que han provocado grandes consecuencias en la humanidad y los agroecosistemas. Contrario a la agricultura convencional, la agricultura orgánica es un sistema que consiste en la utilización de prácticas amigables con el medio ambiente, esta trata de imitar en todo lo posible a la naturaleza.

En Nicaragua el conocimiento de los insectos asociados al cultivo de marañón es limitado ya que solo se han reportado tres especies de insectos plagas entre ellos: el chinche patas de hoja (Leptoglossus zonatus, Dallas), la abejita negra o congó (Trigona silvestrianun) y los comejenes

(Heterotermas $s p$ ) los cuales pueden ocasionar pérdidas considerables en la producción y daños a la planta y frutos de marañón (CIPRES, 2008).

En nuestro país la diversidad de insectos asociados al marañón no ha sido estudiada por lo que no existen reportes que con certeza indiquen su identidad taxonómica. El propósito principal de este estudio fue identificar taxonómicamente, describir la fluctuación poblacional y el rol de los insectos asociados al cultivo de marañón tanto en parcelas orgánicas como en convencionales.

\section{MATERIALES Y MÉTODOS}

Localización de la zona de estudio. El estudio se desarrolló de julio del 2009 a marzo 2010 en la comarca Chacraseca, del departamento de León, esta se encuentra entre las coordenadas $12^{\circ} 26^{\prime}, 58^{\prime \prime}$ de latitud norte y $86^{\circ} 53^{\prime}, 52^{\prime \prime} \mathrm{de}$ longitud oeste, con altura de $109.21 \mathrm{msnm}$. El clima de la zona es cálido los meses más calurosos son Marzo y Abril. La temperatura media anual es de $27^{\circ} \mathrm{C}$, las precipitaciones promedios anuales son de 1300 a $1600 \mathrm{~mm}$ (INETER, 2009).

Descripción de las parcelas en estudio. Para la realización de este estudio, se seleccionaron seis parcelas ya establecidas en tres fincas con manejo orgánico y tres fincas con manejo convencional. En cada parcela se seleccionaron cinco puntos fijos en los que se realizaron muestreos estratificados en cada árbol.

Variables evaluadas en el estudio. Número de insectos Homóptera por familia, número insectos Heteróptera por familia, número de insectos Coleóptera por familia, número de insectos Díptera por familia, y número de insectos Hymenóptera por familia.

Muestreo y recolecta de insectos en el campo. Para realizar el muestreo de insectos en el campo, en cada finca seleccionada, se escogió una parcela de aproximadamente $1 \mathrm{mz}$ (0.7 ha), en esta parcela se seleccionaron 5 sitios de muestreo, cada sitio estaba compuesto por dos árboles de marañón, cada árbol se dividió en tres estratos (parte superior, media e inferior), en cada estrato se colectaron insectos que se observaban en hojas, tallos y estructuras reproductivas (flores y frutos). La captura del espécimen fue de forma manual, estos se conservaron en viales con alcohol al 75\% y fueron llevados al laboratorio en termos con hielo para su posterior identificación.

Descripción del rol de los insectos. Cuando nos referimos al rol, nos referimos a la función o papel que realizan los insectos en el agroecosistema; en las parcelas de marañón, se describió a través de observaciones visuales al momento de los muestreos, así como también información propia que conoce el productor en su finca.

Procesamiento de muestras e identificación de insectos a nivel de laboratorio. El procesamiento de las muestras de insectos en el laboratorio consistió inicialmente en sacar el espécimen de los vasos colectores por fecha y por parcela, estos se vaciaron individualmente sobre papel absorbente, posteriormente, con ayuda de pinceles (tamaño No. 2 de pelo de camello) se realizó la separación de los insectos capturados en grupos para evitar equivocaciones o mezclas de muestras al momento del montaje y la identificación. Para el montaje de los especímenes se utilizaron alfileres entomológicos 
(MORPHO de $4 \mathrm{~cm}$ de longitud), para la identificación de los especímenes se utilizó estereoscopios (CARL ZEISS, modelo 475002 y $475002-9902$ de $4 x, 6.3 x$ y $2.5 x$ ), donde se examinaron las principales características morfológicas de cada insecto colectado. La identificación hasta nivel de orden y familia, se realizó en el laboratorio de entomología y plagas forestales de la UNA; para la identificación hasta familia se utilizaron claves taxonómicas dicotómicas propuestas por Nunes y Dávila (2004). Una vez identificados a nivel de familia, se procedió a la identificación hasta el taxón de géneros y especie. Para ello, se le enviaron los especímenes al museo entomológico de la UNA, en el museo se hicieron comparaciones entre especímenes y se le pasaron claves dicotómicas morfológicas, además, a los insectos se le tomaron fotografías para posteriormente ser enviadas a expertos taxónomos internacionales para su confirmación.
Análisis estadísticos de los datos. Los datos promedios de cada variable (número de insectos), encontrados en cada parcela, fueron comparados por medio de un análisis de varianza (ANDEVA) y una separación de medias mediante la prueba Fisher $(\alpha=0.05)$ en INFOSTAT versión 2009.

\section{RESULTADOS Y DISCUSIÓN}

Familias, géneros y especies de insectos asociados al marañón. En este estudio los principales insectos encontrados en el marañón se presentan en el tabla 1. De acuerdo a nuestras observaciones y a observaciones de los productores, estos son los insectos que más se encontraron en el cultivo.

Tabla 1. Especies de insectos asociados al cultivo de marañón

\begin{tabular}{|c|c|c|c|}
\hline Especie & $\begin{array}{l}\text { Clasificación } \\
\text { taxonómica }\end{array}$ & $\begin{array}{l}\text { Nombre } \\
\text { común }\end{array}$ & $\begin{array}{l}\text { Hábito } \\
\text { alimenticio }\end{array}$ \\
\hline Oncometopia claricor (Walker, 1851) & Homóptera: Cicadellidae & Salta hojas & Chupador \\
\hline Cyrthodisca major (Signoret, 1854) & Homóptera: Cicadellidae & Salta hojas & Chupador \\
\hline Leptoglossus zonatus (Dallas, 1852) & Heteróptera : Coreidae & Chinche patón & Chupador \\
\hline Acanthocephala femorata (Fabricius, 1775) & Heteróptera : Coreidae & Chinche negro & Chupador \\
\hline Hypselonotus lineatus (Stal, 1862) & Heteróptera : Coreidae & Chinche & Chupador \\
\hline Hypselonotus concinnus (Dallas, 1852) & Heteróptera : Coreidae & Chinche & Chupador \\
\hline Epitragus sallei (Champion, 1984) & Coleóptera: Tenebrionidae & Falso alambre & Masticador \\
\hline Lobometopon cupreum (Champion, 1984) & Coleóptera: Tenebrionidae & Falso alambre & Masticador \\
\hline Pantomorus femoratus (Sharp,1891) & Coleóptera: Curculionidae & picudo & Masticador \\
\hline Aulacoscelis tibialis (Jacoby, 1888) & Coleóptera: Chrysomelidae & Mariquita & Masticador \\
\hline Calligarfa sp & Coleóptera: Chrysomelidae & Mariquita & Masticador \\
\hline Charidotellas expunctata (Fabricius, 1781) & Coleóptera: Chrysomelidae & Tortugilla & Masticador \\
\hline Physonota alutacea (Boheman, 1854) & Coleóptera: Chrysomelidae & Tortuguilla & Masticador \\
\hline Hyperaspis jocosa (Mulsant, 1850) & Coleoptera: Coccinélidae & Mariquita & Depredador \\
\hline Aulacosce listibialis (Jacoby, 1888) & Coleoptera: Coccinélidae & --------- & Masticador \\
\hline Cycloneda sanguinea (Linnaeus, 1763 ) & Coleoptera: Coccinélidae & Mariquita & Depredador \\
\hline Chrysopa sp & Neuróptera: Chrysopidae & León de áfidos & Depredador \\
\hline Condylostylus sp & Díptera: Dolichopodidae & Mosquita verde & Depredador \\
\hline Laphria $\mathrm{sp}$ & Díptera: Asilidae & ---- & ---- \\
\hline Trigona silvestrianum (Vachal) & Hymenoptera: Apidae & Abeja congó & Masticador \\
\hline Zacryptocerus multispinus (Emery, 1984) & Hymenóptera: Formícidae & Hormiga & Depredador \\
\hline Dolychoderus $\mathrm{sp}$ & Hymenóptera: Formícidae & Hormiga & Depredador \\
\hline Ectatomma tuberculatum (Oliver, 1791) & Hymenóptera: Formícidae & Hormiga & Depredador \\
\hline Acromyrm exechinato & Hymenóptera: Formícidae & Zompopo & Depredador \\
\hline Polybia occidentalis (Oliver, 1791) & Hymenóptera: Vespidae & Avispa & Polinizador \\
\hline
\end{tabular}

Nota: (Las familias de estos insectos fueron identificadas por el autor de esta investigación, los géneros

y especies fueron identificados por el Ing. Oswaldo Rodríguez, responsable del museo entomológico de la UNA). 
Insectos plagas $y$ benéficos en manejo orgánico $y$ convencional. Para efectos de este estudio y del análisis estadístico realizado, los insectos considerados por el autor y por las observaciones de los productores como plagas y benéficos son: Oncometopia claricor, Cyrthodisca major, Leptoglossus zonatus, Acanthocephala femorata, Epitragus sallei, Lobometopon cupreum, Pantomorus femoratus, Aulacoscelis tibialis, Calligarfa sp, Trigona silvestrianum y los insectos benéficos son: Hyperaspis jocosa, Cycloneda sanguínea, Chrysopa sp, Condylostylus sp, Zacryptocerusmultispinus, Dolychoderus sp, Ectatomma tuberculatum, Polybia occidentalis. Se comparó el número total de insectos plagas e insectos benéficos encontrados en fincas de marañón con manejo orgánico y convencional. El número total de insectos plagas fue mayor en las fincas con manejo orgánico con 1,345 insectos totales, comparado con las fincas con manejo convencional que presentó 984 insectos en total. Cuando se comparan los insectos benéficos, se encontró un mayor número de insectos en las fincas con manejo orgánico con 777 insectos totales, comparado con las fincas con manejo convencional donde se encontró un menor número de insectos benéficos con 719 en total. Se realizó un análisis de varianza del promedio de insectos plagas y benéficos entre las fincas orgánicas y convencionales, para este análisis, se encontraron diferencias significativas $(P=0.0001)$, donde el mayor número de insectos plagas lo presentó el sistema orgánico con promedios de 13.6 insectos por árbol, comparado con el sistema convencional, que presentó promedios de 4.4insectos por árbol. Por el contrario, al comparar el promedio de insectos benéficos en ambas fincas, no se encontró diferencias significativas.

De manera general podemos mencionar que una de las principales razones del porque en las parcelas orgánicas se encontró mayor cantidad de insectos plagas, es debido a que estas fincas no están sometidas a aplicaciones de productos químicos sintéticos por parte de los productores, es posible que la diversidad de insectos en este tipo de manejo, no sufren de un gran impacto en sus poblaciones, probablemente, este puede ser un factor que justifica el haber encontrado mayor cantidad de insectos plagas en este tipo de sistema. También es importante señalar, que en este tipo de fincas orgánicas los productores asocian el cultivo de marañón con otros cultivos tales como cucurbitáceas, frijol, maíz y ajonjolí, esto probablemente ayuda a que haya una mayor diversidad de plantas y por lo tanto mayor cantidad de material vegetal o masa arbórea disponible para los insectos plagas en las fincas orgánicas. Estos resultados concuerda con lo reportado por Nicholl (1999) quien menciona que la razón de encontrar mayor número de insectos en los sistemas orgánicos se puede atribuir a que estos sistemas posen altas concentraciones de biomasa disponible para los insectos fitófagos. En el presente estudio también se observó que la población total de insectos benéficos fue un poco mayor aunque sin diferencia estadística, en las fincas orgánicas esto puede ser posible a que en estos sistemas de fincas hay probablemente una mayor cantidad de presas disponibles para los insectos depredadores. Según Mendoza y Gómez, (1987) la razón de encontrar similares poblaciones de insectos benéficos en ambos tipos de sistemas orgánicos y convencionales, se debe a que los insectos benéficos no exterminan totalmente a sus presas sino que los mantienen a niveles de equilibrio razonables.

Fluctuación poblacional de insectos de la familia Cicadellidae. En la figura 1 se observa que las poblaciones de esta familia de insectos se presentaron en ambos tipos de sistemas en todas las fechas de muestreo excepto en las fechas julio 8 del 2009 y febrero 27 del 2010.De manera general se observa, que el mayor número de estos insectos se presentaron en las fincas con manejo orgánico. En las fincas orgánicas, esta familia de insectos, presentó sus mayores picos poblacional entre las fechas agosto 28 a noviembre 26 del 2009 con 19 y 16 insectos por árbol. En las fincas convencionales, esta familia de insecto presentó los mayores picos poblacionales en las fechas 17 y 22 de diciembre del 2009 con 19 y 9 insectos por árbol. Al realizar el análisis de varianza y separación de medias, para comparar las poblaciones de Cicadellidae entre ambos sistemas de fincas, se encontró diferencias significativas $(P=0.001)$, donde el mayor número de insectos se encontró en las fincas con manejo orgánico con promedios de 1.72 insectos por árbol, que en las fincas con manejo convencional, con promedios de 1.38 insectos por árbol. Pérez (2007) reporta que la gran mayoría de especies de la familia Cicadellidae son de gran importancia agrícola, debido a que actúan como vectores de patógenos entre ellos los virus; el autor de este estudio indica que la alimentación de esta familia de insectos está basada principalmente en savia de hojas y tallos de las plantas.

En este estudio en el cultivo del marañón, de la familia Cicadellidae se encontraron las especies Oncometopia claricor y Cyrthodisca major. No se conoce con exactitud cuál es el rol que juegan estos insectos en este cultivo, sin embargo, al momento del muestreo, se observaron alimentándose de las hojas y brotes de estos árboles, también se observó que estos insectos estaban alimentándose de algunas malezas de hoja ancha. Estos insectos son considerados como plagas por los productores, ya que según sus observaciones, estos aparecen con mucha frecuencia alimentándose de las hojas y brotes del marañón. La presencia de estos insectos se atribuye a que en las fincas orgánicas y convencionales se encontraban una gran diversidad de malezas presentes, estas observaciones coinciden con lo señalado por Sauders (1998) quien indica que la mayoría de los insectos de esta familia se asocian a malezas presentes en los cultivos.

Fluctuación poblacional de insectos de la familia Coreidae. En la figura 2 se observa que las poblaciones de esta familia de insectos se presentaron en ambos tipos de sistemas y en 


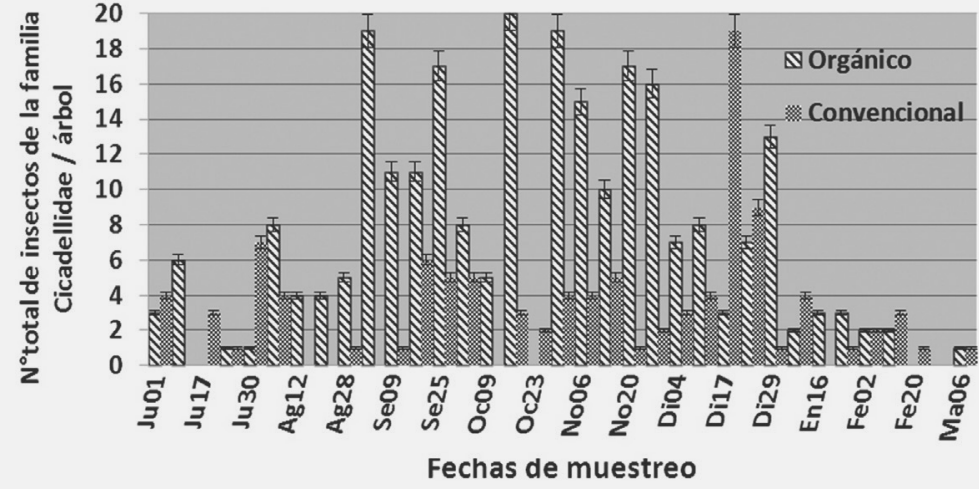

Figura 1. Fluctuación poblacional de insectos de la familia Cicadellidae en el cultivo de marañón orgánico y convencional, julio 2009 a marzo 2010 Chacraseca, León. de marañón es bien conocido ya que esta familia de insectos es específica atacando las semillas de marañón en estado de desarrollo, tanto los adultos como las ninfas succionan el jugo de los frutos y semillas provocando manchas negras y caída de las mismas. Estos insectos son considerados por los productores como una de las principales plagas, ya que causa grandes pérdidas en la producción de semillas de marañón. Durante el muestreo estos insectos se observaron con frecuencia alimentándose de semillas de marañón, también se observaron reproduciéndose en malezas y plantas de marañón.

Creemos que la fluctuación poblacional de esta familia de insectos se debió a que en todas las fechas de muestreo excepto en las fecha diciembre 29 el 2009 y enero 16 del 2010. De manera general se observa que el mayor número de estos insectos se presentaron en las fincas con manejo orgánico que en las fincas convencionales. En las fincas orgánicas, esta familia de insecto, presentó sus mayores picos poblacionales entre las fechas julio 30 y septiembre 09 del 2009 con 24 y 27 insectos por planta. En las fincas con manejo convencional, esta familia de insecto presentó los mayores picos poblacionales entre las fechas julio 01, agosto 12 del 2009 y marzo 06 del 2010 con 8 y 9 insectos por árbol. Al realizar el análisis de varianza y separación de medias para comparar las poblaciones de Coreidae entre ambos sistemas de fincas se encontró diferencias significativas $(P=0.0001)$, donde el mayor número de insectos se encontró en las fincas con manejo orgánico con promedios de 1.8 insectos por árbol, que en las fincas con manejo convencional que presentó promedios de 1.2 insectos por árbol. En este estudio se encontraron los géneros Leptoglossus zonatus, Acanthocephala femorata, Hypselonotus concinnus y Hypselono tuslineatus.

Estudios realizados por Maes (2000) en la Costa Caribe de Nicaragua, también encontró estos géneros asociados a este cultivo. El rol que juegan estos insectos en el cultivo

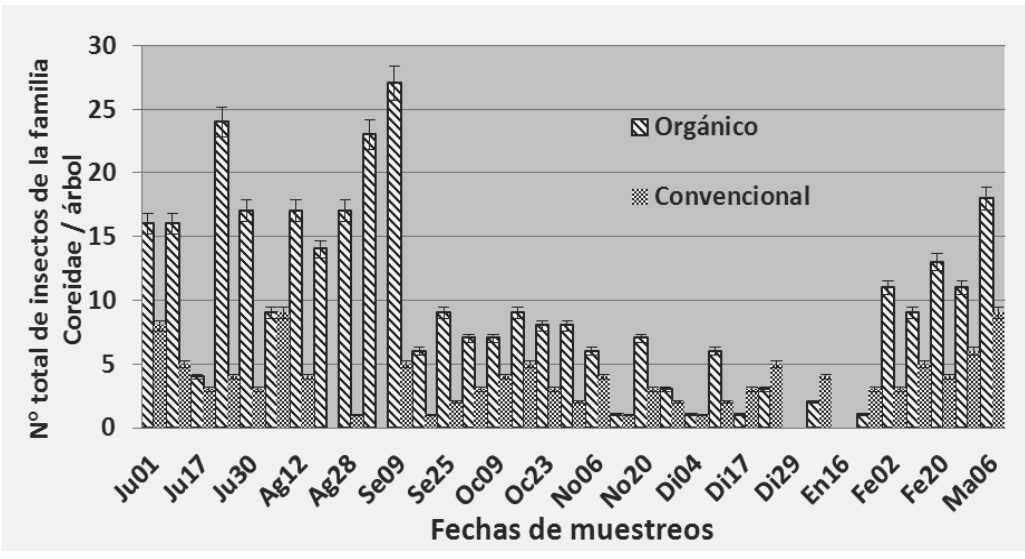

Figura 2. Fluctuación poblacional de insectos de la familia Coreidae en el cultivo de marañón orgánico y convencional, julio 2009 a marzo 2010 Chacraseca, León. esta etapa la cual correspondió a la etapa de crecimiento vegetativo y fructificación, todavía se encontraban árboles con algunos frutos producto de la cosecha anterior, lo que propició una mayor disponibilidad de alimentos para que estos insectos se alimentaran y se reprodujeran.

Fluctuación poblacional de insectos de la familia Tenebrionidae. En esta figura 3 se observa que las poblaciones de esta familia de insectos se presentaron en ambos tipos de sistema en todas las fechas de muestreo excepto en la fecha diciembre 29 del 2009. De manera general se observa, que en ambos sistemas de cultivo el número de insectos se presentó de manera similar, sin embargo, en las fincas convencionales, esta familia de insecto, presentó su mayor pico poblacional en la fecha Julio 30 del 2009 con 18 insectos por árbol. En las fincas orgánicas, esta familia de insecto presentó su mayor pico poblacional en la fecha Julio 08 y Septiembre 17 del 2009 con 24 y 15 insectos por árbol. Al realizar el análisis de varianza y separación de medias, para comparar las poblaciones de Tenebrionidae entre ambos sistemas de fincas, no se encontró diferencias significativas. Según Andrews y Caballero (1989) los adultos y larvas de esta familia se alimentan de raíces, plántulas y semillas. En este estudio se encontró el género Epitragussallei y Lobometoponcupreum. El rol que juegan estos insectos en este cultivo es desconocido, sin embargo, al momento del muestro este insecto se encontró frecuentemente en hojas y yemas de los árboles. Atribuimos esta dinámica dado que en este momento el cual correspondió a la etapa de crecimiento vegetativo, los arboles disponían de una mayor vegetación lo que posiblemente atrajo a más insectos para alimentaran o reproducirse.

Fluctuación poblacional de insectos de la familia Chrysomelidae. En la figura 4 se observa que las poblaciones de esta familia de insectos se presentaron en ambos tipos de sistemas en todas las fechas de muestreo excepto en las 


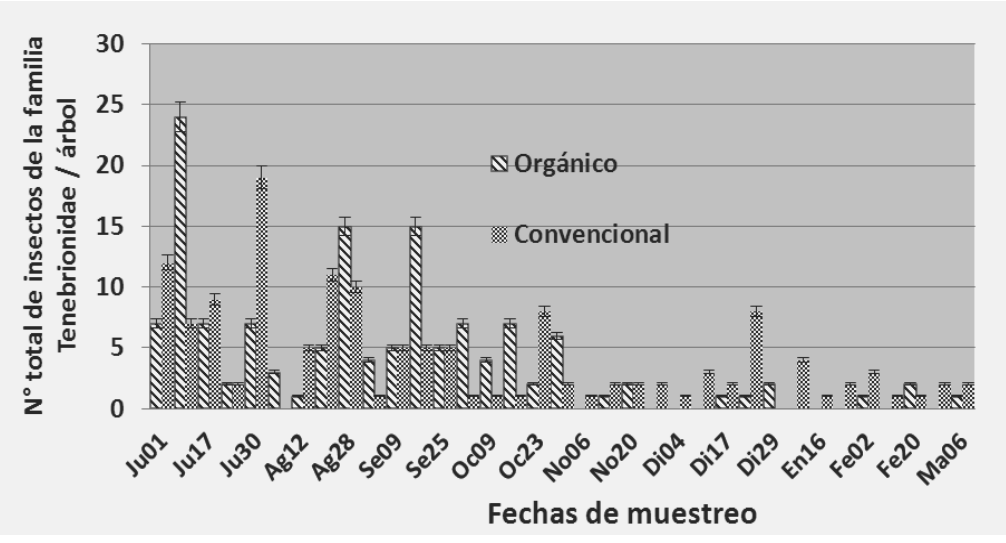

Figura 3. Fluctuación poblacional de insectos de la familia Tenebrionidae en el cultivo de marañón orgánico y convencional, julio 2009 a marzo 2010 Chacraseca, León. árboles. Atribuimos la presencia de estos insectos debió a que en esta etapa los árboles presentaban mayor disponibilidad de follaje para que estos insectos se refugiaran o alimentaran.

Fluctuación poblacional de insectos Hymenoptera de la familia Apidae. La figura 5 , muestra que las poblaciones de esta familia se encontraron con más frecuencia en fincas con manejo orgánico, y las mayores fluctuaciones poblacionales se presentaron entre los meses de enero a marzo 2010 y sus mayores picos poblacionales se presentaron en las fechas febrero 02 y febrero 20 con 72 y 86 insectos por árbol. En las fincas con manejo convencional las poblaciones se presentaron con menos frecuencia $y$ en menor cantidad, sin embargo las mayores fluctuaciones poblacionales se presentaron en la fecha diciembre 17 con 10 insectos por árbol. Al realizar el análisis de varianza y separación de medias para comparar las poblaciones de Apidae entre ambos sistemas de fincas, se encontró diferencias significativas $(P=0.0003)$, donde el mayor número de insectos lo presentó las fincas con manejo orgánico con promedios de 1.75 insectos por árbol, comparado con las fincas con manejo convencional que presentó promedios de 1.06 insectos por árbol. En este estudio se encontró el género Trigona silvestrianum. El rol que juegan estos insectos en marañón es bien conocido dado que estos insectos se alimentan de los falsos frutos y flores para la producción de azucares y mielecillas, esta especie también ataca la semilla o nuez en estado inmaduro (CIPRES, 2008). Durante el muestreo se observó a este insecto alimentándose de los diferentes estados de desarrollo de la semilla así como de los falsos frutos. Atribuimos esta dinámica ya que a partir del mes de Enero se dio el inicio de la floración y fructificación del cultivo, por lo que suponemos que las fluctuaciones poblacionales se debieron a que estos insectos fueron atraídos por la mayor disponibilidad de azucares y miel producidas por las flores y los falsos frutos en estas etapas del cultivo.

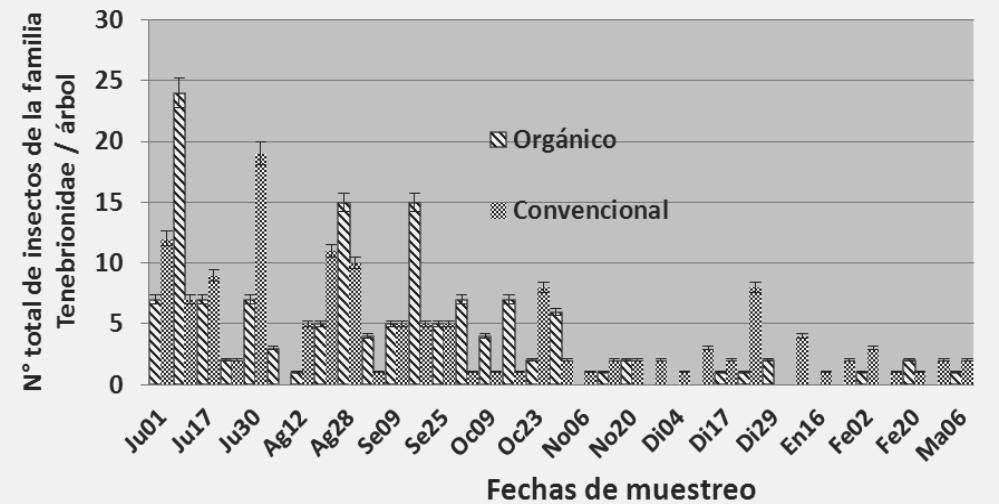

Figura 4. Fluctuación poblacional de insectos de la familia Chrysomelidae en el cultivo de marañón orgánico y convencional, julio 2009 a marzo 2010 en la comarca Chacraseca, León.
Fluctuación poblacional de insectos de la familia Dolichopodidae. En la figura 6 se observa que las poblaciones de esta familia de insectos se presentaron en ambos tipos de sistemas en todas las fechas de muestreo excepto en las fechas julio 30, agosto 12 del 2009 y febrero 02 del 2010. De manera general se observa, que el mayor número de insectos se presentaron en las fincas con manejo orgánico que en las fincas convencionales. En las fincas orgánicas esta familia de insectos presentó sus mayores picos poblacionales entre las fechas noviembre 20 a diciembre 04 del 2009 con 9 y 10 insectos por árbol. En las fincas convencionales, esta familia de insectos presentó los mayores picos poblacionales en las fechas noviembre 20 a 


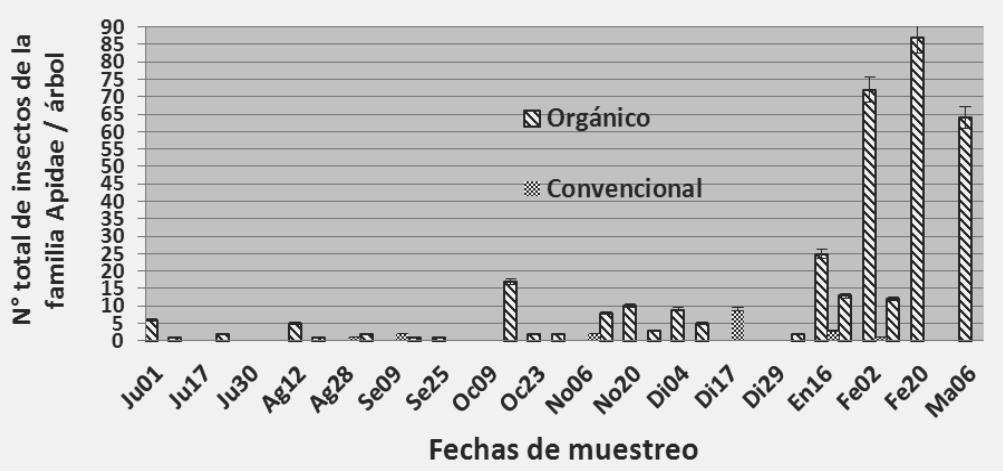

Figura 5. Fluctuación poblacional de insectos de la familia Apidae en el cultivo de marañón orgánico y convencional, julio 2009 a marzo 2010 Chacraseca, León.
02, octubre 09y noviembre 13 del 2009 con 45, 56 y 39 hormigas por árbol. En las fincas con manejo orgánico, esta familia de insecto presentó los mayores picos poblacionales en las fechas octubre 09, octubre 29 del 2009 con 31 y 42 hormigas por árbol. Al realizar el análisis de varianza y separación de medias para comparar las poblaciones de Formicidae entre ambos sistemas de fincas, no se encontró diferencias significativas.

Los géneros encontrados en este estudio fueron: Zacryptocerus multiespinus ,Dolichoderus sp ,Ectatomma, tuberculatum y Acromyrmexechinato. Según perfecto (1994), la gran mayoría de las especies de esta familia son diciembre 04 del 2009con 7 y 9 insectos por árbol. Al realizar el análisis de varianza y separación de medias para comparar las poblaciones de Dolichopodidae en ambos sistemas de fincas, no se encontró diferencias significativas. Según White 2006, los insectos de esta familia juegan un papel importante como agentes de control de plagas, ya que actúan como depredadores de pequeños insectos. En este estudio en el cultivo de marañón, de la familia Dolichopodidae se encontró las especie Condylostylus sp. No se conoce con certeza el rol que juegan estos insectos en marañón, no obstante al momento del muestreo este insecto se encontró con frecuencia en hojas y brotes de las ramas. La dinámica de estos insectos se atribuye a que en las fincas tanto orgánicas como convencionales se encontraban en la etapa de floración, y por tanto había una mayor disponibilidad de refugio y alimento para esta familia de insectos.

Fluctuación poblacional de insectos Hymenoptera de la familia Formícidae. La figura 7 muestra que las mayores fluctuaciones poblacionales se presentaron con mucha frecuencia en ambos sistemas de fincas se presentaron entre los meses de octubre y diciembre 2009, de manera general se observa que el mayor número de estos insectos se presentaron en las fincas con manejo convencional en las fechas octubre

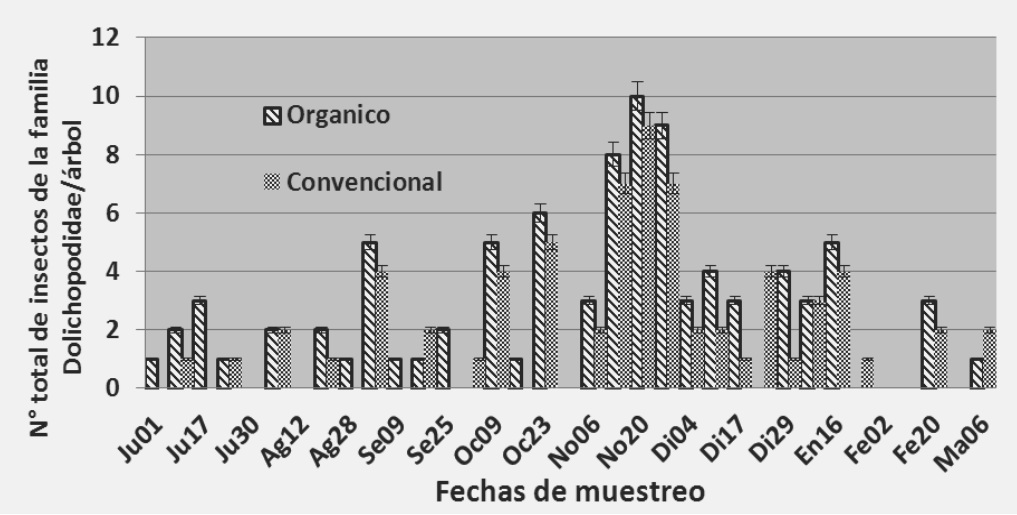

Figura 6. Fluctuación poblacional de insectos de la familia Dolichopodidae en el cultivo de marañón orgánico y convencional, julio 2009 a marzo 2010 Chacraseca. depredadoras generalistas ya que se alimentan de semillas, mielecillas de áfidos e insectos vivos y muertos. En el cultivo de marañón no se conoce aún cual su rol debido a que al momento del muestreo no se observó alimentándose de algún tipo de insecto plaga, sin embargo, estos insectos se observaron con mucha frecuencia en hojas y ramas del cultivo. Creemos que en esta etapa, la cual corresponde a la etapa de mayor crecimiento vegetativo, el cultivo disponía de más presas y hospederos, lo que atrajo a mayores predadores para que estos se alimentaran y reprodujeran.

Fluctuación poblacional de insectos Hymenoptera de la familia Vespidae. En la figura 8 de manera general se observa que en las fincas con manejo orgánico las fluctuaciones poblaciones fueron mayores que en comparación con las fincas con manejo convencional. En las fincas con manejo orgánico los mayores picos poblacionales se presentaron en dos momentos, el primero se presentó entre los meses de agosto a octubre 2009, y el segundo entre los meses de Diciembre 2009 a febrero2010, sin embargo, los mayores picos poblacionales se presentó en las fechas enero $08 \mathrm{y}$ febrero 12 del 2010 con 17 y 28 avispas por árbol. En las fincas con manejo convencional las poblaciones fueron bajas, no obstante el mayor pico poblacional se presentó en las fechas octubre 23 y diciembre 04 del 2009 con 13 avispas por árbol para ambas fechas de muestreo. Al realizar el análisis de varianza y separación de medias para comparar las poblaciones de avispas, entre ambos sistemas de fincas, se encontró diferencias significativas $(P=0.001)$, el mayor número de insectos lo presentaron las fincas con por árbol, comparado con las fincas con manejo convencional que presentaron promedios de 1.3 insectos por planta. En este estudio se encontró el género Polybiaoccidentalis. Los insectos de esta familia juega un papel muy importante ya son insectos depredadores de huevos, larvas, pupas y de otros insectos que son dañinos en los cultivos manejo orgánico con promedios de 1.7 insectos 


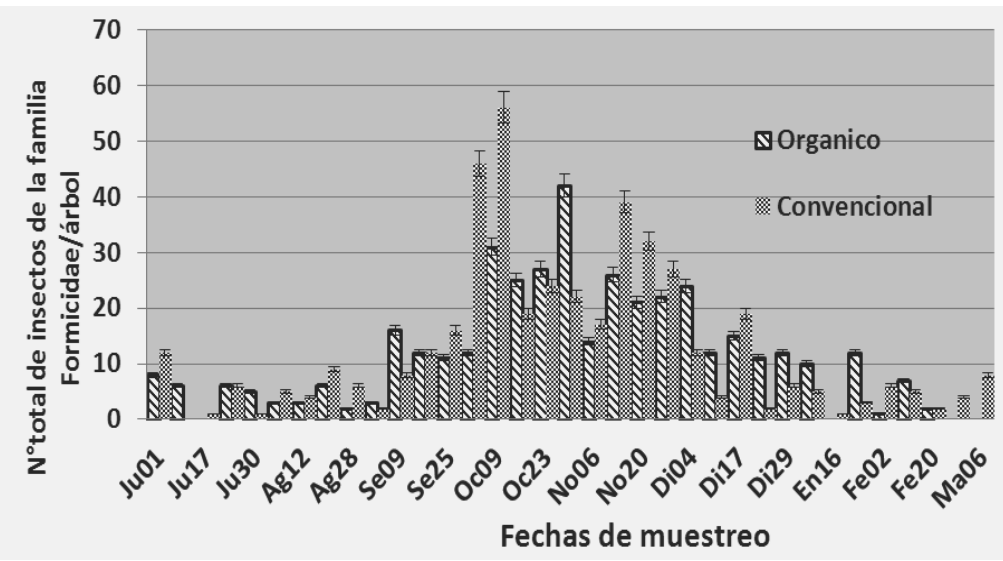

Figura 7. Fluctuación poblacional de insectos de la familia Formicidae en el cultivo de marañón orgánico y convencional, julio 2009 a marzo 2010 Chacraseca, León.

frutales; Según Sáenz y De la Lana (1990) estas especies son polinizadoras, productoras de mielecillas por lo que es común encontrarlas en cultivos con presencia de flores. El rol de estos insectos en marañón fue muy notorio ya que durante el muestreo estos insectos se observaron succionando la mielecilla de las flores y frutos de marañón. Esta dinámica se puede atribuir debido a que en esta etapa el cultivo estaba en plena floración por lo tanto suponemos que en este momento había mayor disponibilidad de presas, polen o mielecillas que estos insectos necesitan para alimentarse y reproducirse. Según Jiménez (2008) el olor o aroma de las flores juegan un papel principal como atrayente de insectos polinizadores en las plantas.

\section{CONCLUSIONES}

Los insectos asociados al marañón son especies miembros de la familias: Coreidae, Cicadellidae, Tenebrionidae, Chrysomelidae, Apidae, Formicidae, Dolichopodidae, y Vespidae.

Los insectos Coreidaey Apidae, se presentaron con más frecuencia durante la época de fructificación del cultivo.

Los insectos de las familias Dolichopodidae y Vespidae, se presentaron con más frecuencia durante la época de floración del cultivo.

Se encontró que las familias Cicadellidae, Tenebrionidae, Chrysomelidae y Formicidae, se presentaron con más frecuencia durante la época de crecimiento vegetativo del cultivo.

Se encontró un mayor número total de insectos plagas y benéficos en las parcelas de marañón con manejo orgánico que en las parcelas convencionales.

Se reporta que las principales insectos plagas asociadas al marañón son el chinche patas de hojas y la mosquita negra.

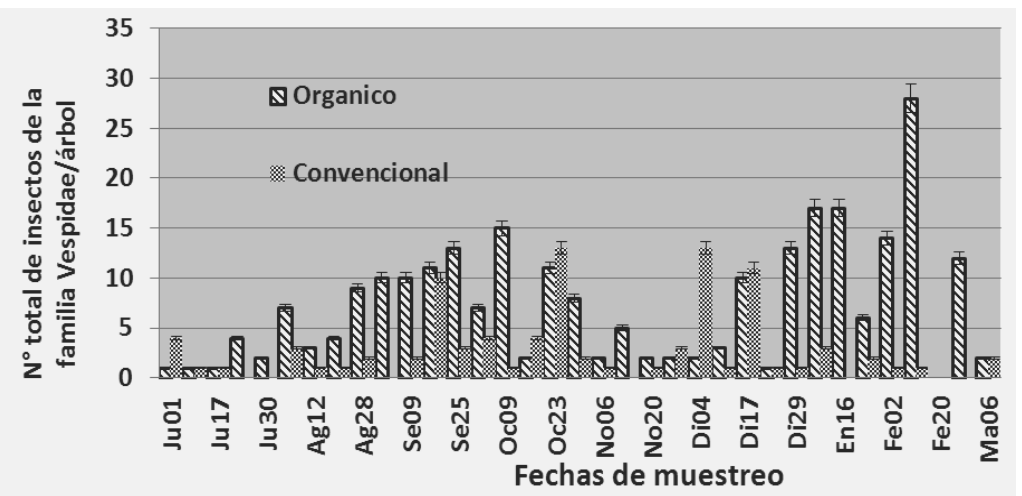

Figura 8. Fluctuación poblacional de insectos de la familia Vespidae en el cultivo de marañón orgánico y convencional, julio 2009 a marzo 2010 en la comarca Chacraseca, León. 


\section{REFERENCIAS BIBLIOGRÁFICAS}

Andrews, K; Caballero, R. 1989. Guía para el estudio de órdenes y familias de insectos de Centroamérica. Escuela Agrícola Panamericana, Zamorano, Honduras. 179 p.

CIPRES (Centro Parea la promoción, la investigación y el desarrollo rural y social). 2008. Guía para el manejo de las principales plagas de marañón orgánico en Nicaragua, 1era edición. 39 p.

Gliessman, R, Stephen. 1998. Agroecología, Procesos Ecológicos en Agricultura Sostenible. CATIE,Turrialba, C R. 380 p.

Guía técnica para el manejo de la nuez de maranón, variedades de injerto CIAL- CRM, 2007. Cuenta reto del milenio. 64 p.

INETER, 2009. Instituto Nicaragüense de Estudios Territoriales de Nicaragua. Dirección general de meteorología. Resumen meteorológico diario del 2009 y 2010. Managua Nicaragua. 78 p.

InfoStat, 2008. InfoStat, versión 2008. Manual del Usuario. Grupo InfoStat, FCA, Universidad Nacional de Córdoba. Primera Edición, Editorial Brujas Argentina. 334 p.

Jaramillo, L. 2003. Informe. EL MARAÑON (Anacardium. occidentale) 23 p.

Jiménez, E. 2008. Texto básico: Manejo integrado de plagas. Universidad Nacional agraria (UNA), Managua, Nicaragua. 108 p.

León, G. 2005. La diversidad de insectos en cítricos y su importancia en los programas de manejo integrado de plagas. Manejo Integrado de plagas. No 74. p 85 - 93 .

Maes, J, M. 2004. Insectos asociados a algunos cultivos tropicales en el Atlántico de Nicaragua. Museo entomológico. Rev.Nica.Ent. NO 64. Suplemento 1. Parte XII. p 1-64.

Membreño, P. 2002. El Marañón (Anacardium occidentales, L) 28 p.

Mendoza, H.F; Gómez, S.J. 1987. Entomología General, Valdivieso, A.V (Edit) 1ed.Ciudad de la Habana, Cuba, Editorial Pueblo y Education. $226 \mathrm{p}$.

Nicolls, C; Altieri, MA. 1999.Manual práctico de control biológico para una agricultura sostenible. California EUA. 8 p.

Nunes, Z. C, Dávila, A. L. 2004. Taxonomía de las principales familias y subfamilias de insectos agrícolas en Nicaragua. UCATSE. Universidad Católica Agropecuaria del Trópico seco 1era edición Estelí, Nicaragua. 164 p.

Pérez, G. 2007. Evaluación del comportamiento de Oncometopiaclarior (Walker) (Hemíptera: Cicadellidae) ante especies vegetales asociadas al cultivo Dracaenamarginata (Lamarck) y su preferencia a diversos regímenes de fertilización. Tesis Msc. Programa de Educación para el Desarrollo y la Conservación del Centro Agronómico Tropical de Investigación y Enseñanza (CATIE) Turrialba, Costa Rica, 87 p.

Perfecto, I. 1994. El sistema de plagas hormigas- maíz en Nicaragua. Manuales del curso y foro sub-regional centroamericano y del Caribe de control biológico de plagas. León, Nicaragua. Sección VI. Capitulo XXIII, 34 p.

Saenz, M. De la Llana, A. 1990. Entomología Sistemática. UNA (Universidad Nacional Agraria). Managua, Nicaragua. 225 p.

White, R. 2006. Peterson field guides beetles: a field guide to the beetles of north America. Sistematic Entomology Laboratory U.S. Departament of Agriculture. 368 p. 